TITLE:

\title{
Shape dependent thermal effects in apertured fiber probes for scanning near-field optical microscopy
}

\section{$\operatorname{AUTHOR}(\mathrm{S}):$}

Ambrosio, A; Fenwick, O; Cacialli, F; Micheletto, R; Kawakami, Y; Gucciardi, PG; Kang, DJ; Allegrini, M

\section{CITATION:}

Ambrosio, A ... [et al]. Shape dependent thermal effects in apertured fiber probes for scanning near-field optical microscopy. JOURNAL OF APPLIED PHYSICS 2006, 99(8): 084303.

\section{ISSUE DATE:}

2006-04-15

URL:

http://hdl.handle.net/2433/50156

\section{RIGHT:}

Copyright 2006 American Institute of Physics. This article may be downloaded for personal use only. Any other use requires prior permission of the author and the American Institute of Physics. 


\title{
Shape dependent thermal effects in apertured fiber probes for scanning near-field optical microscopy
}

\author{
A. Ambrosio, ${ }^{\text {a) }}$ O. Fenwick, and F. Cacialli ${ }^{\text {b) }}$ \\ Department of Physics and Astronomy, University College London, Gower Street, WC1E 6BT London, \\ United Kingdom and London Centre for Nanotechnology, University College London, 2-16 Torrington Place, \\ WC1E $7 H N$ London, United Kingdom \\ R. Micheletto and Y. Kawakami \\ Department of Electronic Science, Graduate School of Engineering, Kyoto University, Nishikyo-ku, \\ Katsura, 615-8510 Kyoto, Japan \\ P. G. Gucciardi \\ CNR-Istituto per i Processi Chimico-Fisici, Sezione di Messina, Via La Farina 237, I-98123 Messina, Italy \\ D. J. Kang \\ Nanoscience, University of Cambridge, 11 J.J. Thomson Avenue, CB3 OFF Cambridge, United Kingdom; \\ Sungkyunkwan Advanced Institute of Nanotechnology, Sungkyunkwan University, Suwon 440-746, \\ South Korea; and Department of Physics, Sungkyunkwan University, Suwon 440-746, South Korea

\section{Allegrini} \\ Dipartimento di Fisica "Enrico Fermi," Università di Pisa, Largo Bruno Pontecorvo 3, I-56127 Pisa, Italy \\ and CNR-INFM polyLab, Largo Bruno Pontecorvo 3, I-56127 Pisa, Italy
}

(Received 28 September 2005; accepted 26 February 2006; published online 18 April 2006)

Metal-coated, "pulled," and conically shaped fiber probes used in scanning near-field optical microscopy (SNOM) typically undergo a thermal expansion when injected with laser light, due to partial energy absorption by the metallic film. Here, we report investigations into the thermal behavior of fiber probes produced by selective chemical etching that in our experience provide high light throughputs $\left(10^{-3}-10^{-4}\right.$ vs $10^{-6}$ for the pulled fibers). Unexpectedly, we find a shortening of such probes in response to "high-power" laser injection $(>1 \mathrm{~mW})$. Thermal stress due to prolonged high-power laser injection $(\sim 9 \mathrm{~mW}$ at $325 \mathrm{~nm}$; compared to powers $<1 \mathrm{~mW}$ often used in SNOM experiments) determines permanent alterations of the probes, after which their thermomechanical behavior reverts to the commonly observed elongation in response to laser injection. Scanning electron microscopy after high-power irradiation on such probes shows partial detachment of the metallic coating near the fiber termination. This, however, does not appear to compromise the probe's performance in terms of light confinement outside the aperture area, suggesting that the detachment only affects the coating over the fiber cladding and confirming the operational robustness of these probes. In comparison, tube-etched, conical probes display substantial damage of the coating, up to several microns from the apex, after being injected with a comparable high-power laser beam $(>10 \mathrm{~mW}$ at $633 \mathrm{~nm})$. Although the vertical feedback mechanism of the microscope can compensate for dilations/contractions of the probes, these findings are of general importance to the field. More specifically they are significant for the achievement of a detailed understanding of apertured-SNOM operation, for the selection and operation of near-field probes, and for preventing potential artifacts in imaging and lithography, due to uncontrolled alteration of the probe properties and/or light leakage from cracks of the opaque coating induced by thermal fatigue. In addition, our results demonstrate that it is important for probe design to also consider the probe's thermal regime during operation, so as to prevent cracks in the functional parts of the coating and thus spurious, undesired sample illumination from regions other than the probe intended aperture. (c) 2006 American Institute of Physics. [DOI: 10.1063/1.2188250]

\section{INTRODUCTION}

Scanning near-field optical microscopy ${ }^{1,2}$ (SNOM) is a technique capable of extending the spatial resolution of optical experiments down to the nanometer scale. SNOM overcomes the diffraction limit by scanning a nanometric probe

\footnotetext{
${ }^{a}$ Current address: Dipartimento di Fisica, Università di Pisa; electronic mail antonio.ambrosio@df.unipi.it

b)Electronic mail: f.cacialli@ucl.ac.uk
}

close to the sample surface. The probe can be used to either illuminate locally the surface, ${ }^{1}$ collect the radiation scattered by the sample, ${ }^{3}$ or both. ${ }^{4}$ The so-called aperture SNOM commonly exploits optical fiber sensors ${ }^{5}$ featuring an apical aperture with diameters in the $20-200 \mathrm{~nm}$ range. The fabrication process consists of three steps: first, one of the ends of the optical fiber is tapered, then coated with an opaque (metallic) film, and finally an aperture is defined at the cone apex. Tapering is accomplished by either heat and pull, ${ }^{5-7}$ or 
by chemical etching methods. ${ }^{8-10}$ Here, double- and tripletapering techniques ${ }^{4,11}$ as well as dynamic etching ${ }^{12}$ have been introduced to increase the cone angle, and with it the light throughput. Several procedures have also been developed to form the apertures. The most common technique involves deposition of a metal coating onto the tapered region at a steep angle, which produces a metal-free region at the very end of the tip. ${ }^{13}$ Alternatively, sub-100-nm apertures can be formed by hard tapping, ${ }^{1,4}$ selective resin coating, ${ }^{14-16}$ focused ion beam milling, ${ }^{17}$ and solid state electrolysis. ${ }^{18}$

Typical metals used for coating the probe sidewalls are gold, silver, or aluminum, often preceded by a thin chromium film to enhance adhesion. The thickness of the metallic film $(\sim 100 \mathrm{~nm})$ is chosen to be much greater than the skin depth of the material at the specific working wavelength. The waveguide properties of such complex optical systems, involving both a dielectric core and the metallic coating, have been studied by several groups (see Refs. 19-21 and references therein). Multiple reflections at the metallized sides $^{19,22}$ are responsible for the confinement of the light towards the apical aperture. Such reflections have a twofold effect. First, they reduce the throughput of the probes to $10^{-6}-10^{-3}$ since no other solution to Maxwell's equations other than HE11 exists below a certain fiber radius. ${ }^{20} \mathrm{Sec}$ ond, they also increase the total amount of light absorbed by the metal coating $(20 \%-30 \%$ of the coupled power, to be compared to the $8 \%$ of the aluminum absorption at normal incidence), so that the temperature of the metallic film in the apical region can increase up to several hundreds of degrees. ${ }^{23}$ As a consequence, the probe undergoes a thermal expansion $^{24,25}$ and the light throughput changes. It can either increase $^{26,27}$ or decrease, ${ }^{26}$ depending on the prevalence of the thermal expansion of the aperture, or of the elongation of

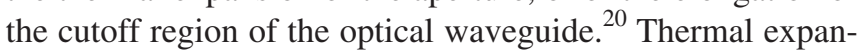
sions up to a few hundreds of $\mathrm{nm} / \mathrm{mW}$ of coupled power have been reported, ${ }^{24}$ occurring on time scales in the millisecond range, with a faster component of a few tens of microseconds. ${ }^{28}$

The study of the probe heating and of heat diffusion mechanisms has important implications. For example, due to the close proximity $(\sim 1-10 \mathrm{~nm})$ of the nanoscopic probe from the sample surface, a significant tip-to-sample heat transfer can occur, ${ }^{29}$ and this could alter the properties of the specimens, with consequences especially in nanolithography $^{30}$ or in shear-force and spectroscopic SNOM imaging. ${ }^{31}$ Although, several papers have been published on the thermal expansion of SNOM fiber probes, ${ }^{22-28}$ the analysis has so far been focused on pulled or etched aluminumcoated probes. Such probes are characterized by a uniform and very long conical taper $(\sim 1 \mathrm{~mm})$ which is uniformly coated.

In this article we show that SNOM probes produced by selective chemical etching (SCE) respond to light injection with a contraction of their overall length in their pristine state. Such thermomechanical behavior is unexpected, since opposite to that observed with "pulled" probes, and we attribute it to the different shapes of the probes produced with the different methods. Note also that although the vertical feedback mechanism of the microscope can compensate for
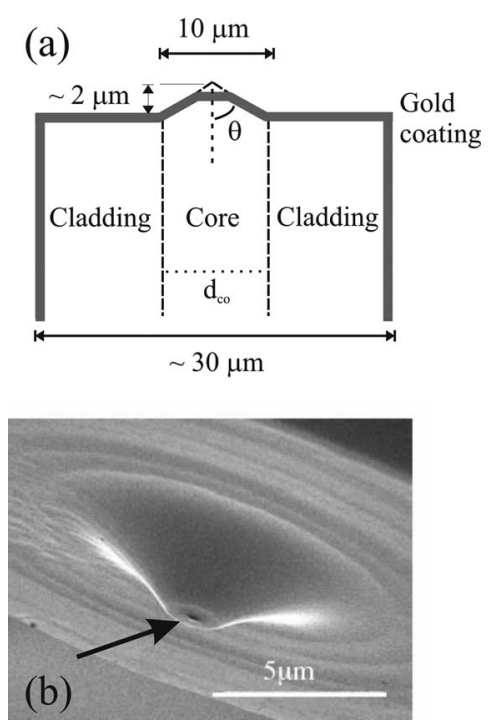

FIG. 1. (a) Schematic of a SNOM fiber probe produced by selective chemical etching (SCE). The protruding cone is formed due to a slower etching rate of the core with respect to the cladding. After gold metallization (indicated by gray lines), the tip is punched against a hard surface, producing a flattened apex with a subwavelength aperture at the center (black arrow), as evidenced by the SEM micrograph in (b).

dilations/contractions of the probes, these findings are of general importance to the advancement of SNOM-probe technology. More specifically they are significant for the achievement of a detailed understanding of apertured-SNOM operation, for the selection and operation of near-field probes and for preventing potential artifacts in imaging and lithography, due to uncontrolled alteration of the probe properties and/or light leakage from cracks of the opaque coating induced by thermal fatigue. In addition, our results demonstrate that it is important for probe design to also consider the probes thermal regime during operation, so as to prevent cracks in the functional parts of the coating and thus spurious, undesired sample illumination from regions other than the probe intended aperture.

\section{EXPERIMENTAL SETUP}

Experiments are carried out on high-throughput fiber probes specially designed for UV near-field spectroscopy ${ }^{32}$ and lithography, ${ }^{33,34}$ purchased from Jasco Corporation. ${ }^{35}$ These sensors are based on quartz fibers (10/125 $\mu \mathrm{m}$ core/ cladding diameters) stripped of the outer polymeric jacket. Tapering is accomplished by means of selective chemical etching in bulk, in a hydrofluoric acid solution ( $\mathrm{HF}, 50 \%$ ) buffered with ammonium fluoride $\left(\mathrm{NH}_{4} \mathrm{~F}\right){ }^{8}$ Such etching procedure results in probes terminations that are significantly different to those produced by pulling or tube etching, which end instead with a long conical taper $(0.1-1 \mathrm{~mm})$. As depicted in Fig. 1(a), the fiber end of SCE probes is characterized by a glass cylinder a few tens of microns wide, with a short cone (only a few microns long) protruding from the cylinder base. The tip-cone angle is controlled by adjusting the temperature of the solution, the etching time, and the chemical composition of the solution. ${ }^{14}$ The volume ratio of $\mathrm{NH}_{4} \mathrm{~F}$ influences the relative core/cladding etching rates $\left(r_{\text {co }}\right.$ 


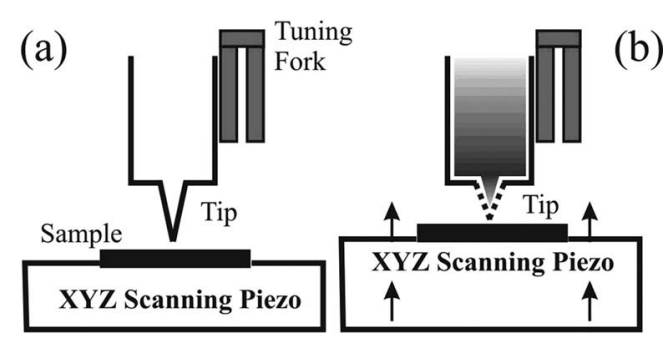

(c)
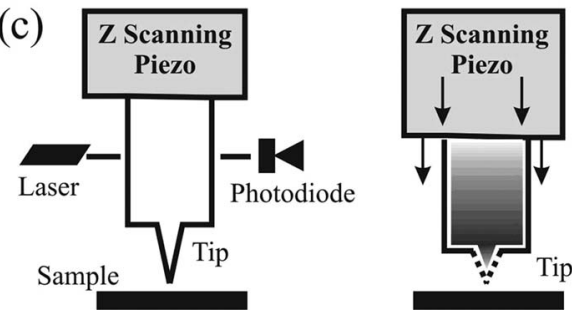

(d)

FIG. 2. Sketch of the setup to measure tip thermomechanical response Before coupling light into the probe, the tip is approached to the sample, and the mutual distance stabilized by means of a feedback circuit driven by nonoptical (a) or optical (c) shear-force detection. The light-induced tip shortening $\Delta h_{\text {tip }}$ is compensated by the feedback loop which will move the sample up (b) or the tip down (d) of the same amount.

and $r_{\mathrm{cl}}$, respectively). These parameters, according to Ref. 36 , define the cone height $h_{\text {tip }}$ and angle $\theta$ as

$$
\begin{aligned}
& h_{\mathrm{tip}}=\frac{d_{\mathrm{co}}\left(r_{\mathrm{cl}}-r_{\mathrm{co}}\right)}{2 r_{\mathrm{co}}}, \\
& \theta=\frac{r_{\mathrm{co}}}{\left(r_{\mathrm{cl}}-r_{\mathrm{co}}\right)},
\end{aligned}
$$

where $d_{\mathrm{co}}$ is the core diameter and $\theta$ is in radians. The Jasco NPU-series probes are characterized by cone lengths of $\sim 2 \mu \mathrm{m}$, corresponding to angles of $\sim 45^{\circ}$, as shown in Fig. 1(a). The cladding is only partially etched, and its residual diameter ranges from 25 to $50 \mu \mathrm{m}$. A gold film $\sim 150 \mathrm{~nm}$ thick (light gray line) is sputtered on the entire exterior surface. The small aperture is subsequently obtained by pushing the probe against a flat surface, squeezing the gold off the side while controlling the light throughput. ${ }^{4}$ The scanning electron microscope (SEM) picture in Fig. 1(b) evidences that the resulting conical tip shows a flat plane, orthogonal to the fiber axis, with a round aperture at the center. The optical and topographic interaction of the probe with the sample surface occurs via the protrusion indicated by the arrow.

To investigate thermal expansion effects in such probes we used the method described in Ref. 25. The tip is first brought in close proximity of the sample surface, stabilizing the tip-sample distance by means of a shear-force driven feedback loop [Fig. 2(a)]. A controlled amount of light (HeCd laser, $\lambda=325 \mathrm{~nm}, P$ up to $9 \mathrm{~mW}$ ) is thus sent into the fiber launching assembly. Due to light absorption, the probe heats up, and as a result of thermal dilation of the metallic coating its length varies by an amount $\Delta h_{\text {tip }}$. To counterbalance this effect and restore the preset tip-sample distance, the feedback loop moves the sample of the same amount $\Delta h_{\text {tip }}$ [Fig. 2(b)]. The thermomechanical response of the probe (either elongation or shortening) can thus be measured by

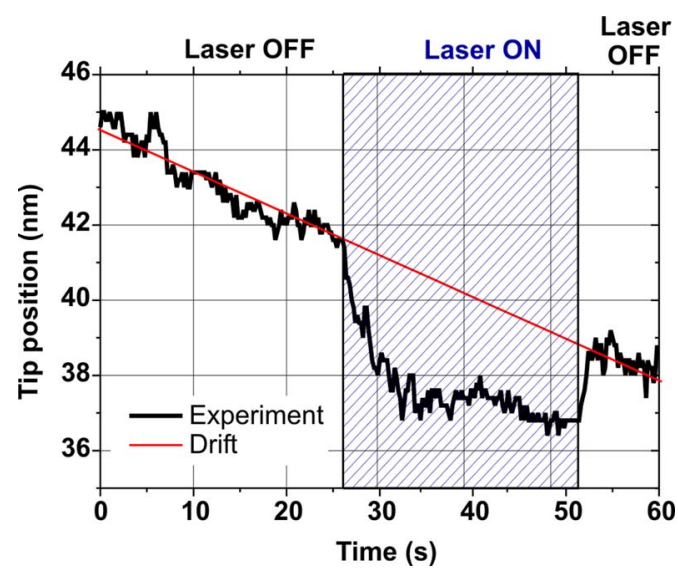

FIG. 3. (Color online) Plot of the probe position as a function of time (black line). When light is coupled (boxed region) the probe is pushed downward as a response to the tip shortening. Cutting out the laser beam, the probe is lifted up. The effect is clearly distinguishable from the mechanical drift of $\sim 0.1 \mathrm{~nm} / \mathrm{s}$ (red line).

monitoring the error voltage provided by the feedback circuit as a function of the coupled light. Experiments are carried out on two different apparatuses. Measurements of the tip length variations are accomplished with a homemade SNOM (Ref. 33) with nonoptical shear-force detection, ${ }^{37}$ in which the sample is scanned by means of a piezoelectric translation stage (PI P562-3CD). An ADW in digital signal processing board is exploited to drive the feedback loop, to set the scanning voltages, and to acquire the feedback signal. We use a digital oscilloscope to monitor the $z$-control output of the feedback. In addition, an opportunely modified ${ }^{38}$ commercial SNOM (Unisoku Ltd., Japan) is used to follow the tip length variation as a function of the time, when the light is coupled into the sensor. This apparatus employs an optical shearforce detection scheme ${ }^{39}$ to control the tip-sample distance [Fig. 2(c)]. A set of three piezotubes (not shown in the figure) scans the sample in the $x y$ plane $\left(15 \times 15 \mu \mathrm{m}^{2}\right.$ travel distance). A further piezotube is exploited to move the tip vertically (scan range of $6 \mu \mathrm{m}$ ), as shown in Fig. 2(d). A digital oscilloscope is used to monitor the movement of the piezo holding the tip.

\section{RESULTS AND DISCUSSION}

We start by reporting investigations carried out with the help of the setup in Figs. 2(c) and 2(d). For these we used new probes, as received from Jasco, and never used for scanning. Figure 3 (black line) shows the temporal evolution of the vertical position of the tip, during an off-on-off cycle of laser illumination, in which we observe a continuous downward drift of the tip $(\sim 0.1 \mathrm{~nm} / \mathrm{s}$, red line $)$ due to minor mechanical or electrical instabilities of the SNOM apparatus that is particularly obvious in the off period. The most remarkable feature of Fig. 3, however, is the downward shift of the tip position $(4 \mathrm{~nm})$ that takes place at the beginning of the on period. After the laser is switched off, the piezo retracts the tip by $\sim 2 \mathrm{~nm}$ (note that linear interpolation of the drift during the off period suggests a $2.5 \mathrm{~nm}$ "intrinsic" downward shift of the SNOM over the same period). Such a behavior is opposite to the one expected for thermal elonga- 


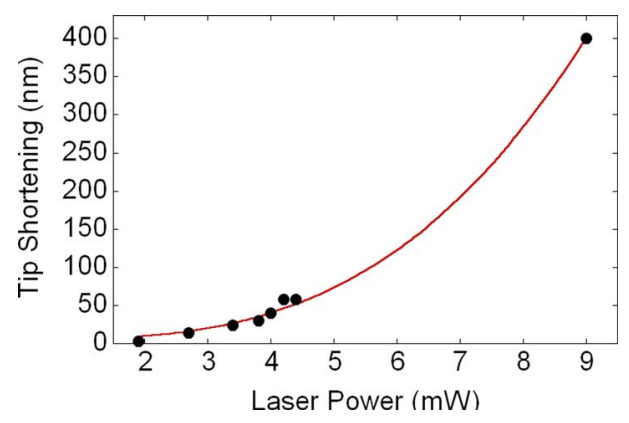

FIG. 4. (Color online) Plot of the tip shortening measured vs laser power (black circles). Injection losses are typically $90 \%$. The nonlinear trend is fitted by a cubic law (red line).

tion of the probe that would have forced an upward shift of the tip/piezo system and thus points instead to an overall shortening of the glass-metal assembly [Fig. 2(c) and 2(d)].

We then used the setup in Figs. 2(a) and 2(b) to analyze the dependence of such effect on the power coupled into the fiber and report the results in Fig. 4. For this experiment we mounted another new probe (in the following addressed as probe A) and modulated the laser power by means of a shutter (100\% modulation depth, duty cycle of several seconds) while simultaneously monitoring the feedback signal to the piezostage (i.e., the one controlling the vertical movements of the sample) by means of a digital oscilloscope. We were thus able to observe very significant tip shortenings, up to $400 \mathrm{~nm}$ for a laser power $\mathrm{P}_{\text {las }}=9.2 \mathrm{~mW}$ (note that this was the power before coupling into the fiber and that we estimate injection losses to be greater than $50 \%$ and possibly up to $90 \%)$. As evident from Fig. 4, the power dependence of this effect is highly nonlinear, and we find that the experimental data can be fitted by a cubic model $\Delta h_{\text {tip }} \propto P_{\text {las }}{ }^{3}$ We have also noted that especially at the highest powers the shortening effect decreases with successive cycles of irradiation. For example, it has not been possible to recover the original value of $400 \mathrm{~nm}$ after leaving the system running for a long period of time (about $4 \mathrm{~h}$ ). In addition, after some minutes of laser irradiation at $9 \mathrm{~mW}$ we recorded a sudden event that led to fast tip retraction, and after which we could no longer observe a photoinduced shortening of the tip. Such observations suggest that some permanent alteration of probe A had occurred, although we could not observe any luminous emission from the tip apex, which is the usual sign of tip degradation. Such behavior also confirms our experience that these probes are robust and durable (incidentally, we note that Jasco believes that the maximum input tolerance of near-field products is $1-2 \mathrm{~mW}) .^{40}$

Perhaps the most interesting aspect of the phenomenon is that such modification is accompanied by a radical change of the thermomechanical behavior of the probe that now begins to elongate in response to laser injection. For example, we measured an elongation $\Delta h_{\text {tip }} \sim 5 \mathrm{~nm}$ for $P_{\text {las }}=9 \mathrm{~mW}$ at $325 \mathrm{~nm}$. When changing the wavelength to $633 \mathrm{~nm}$, we recover the same behavior, with $\sim 2 \mathrm{~nm}$ elongation. Incidentally similar observations were obtained with an old probe (probe $\mathrm{B}$ in the following) that had already been used for scanning on polymers films, so intensively, however, that light could be observed emerging from the tip apex even by

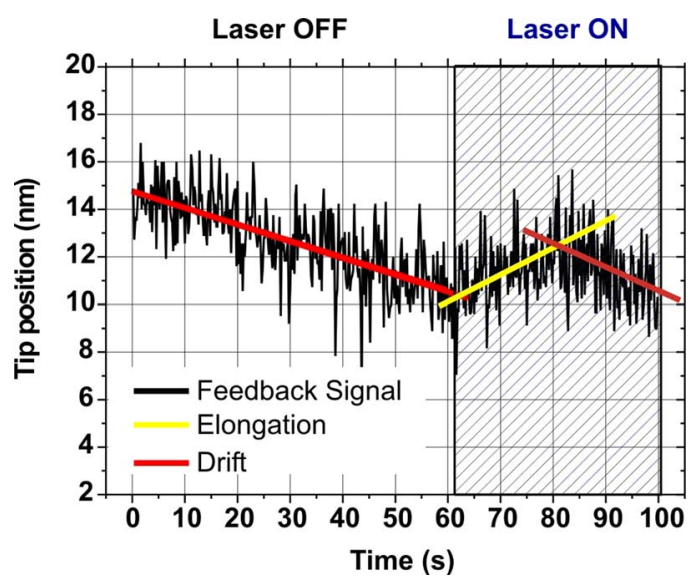

FIG. 5. (Color online) Thermomechanical behavior of an intensively used probe (black line). When light is coupled into the fiber (boxed region), the probe is lifted up from the sample surface by the feedback loop (yellow line), in response to the thermal elongation of the tip. The effect is superimposed to a mechanical drift (red line) of the order of $0.07 \mathrm{~nm} / \mathrm{s}$. Data in this plot were obtained with the setup described in Figs. 2(c) and 2(d).

the naked eye, indicating certain damage to the metallic coating defining the aperture. The thermal behavior of probe B when subjected to laser irradiation is reported in Fig. 5. Superimposed to the mechanical drift (red line, $\sim 0.07 \mathrm{~nm} / \mathrm{s}$ ), we note that the feedback lifts up the tip when we switch the laser on (boxed region): the clear fingerprint of a thermal elongation. Finally, we observe that the thermal elongation occurs on longer time scales, suggesting that a different process is taking place.

Figure 6 shows the scanning electron microscope (SEM) picture of probe A after the alteration has occurred. The appearance of the end cone is very different from the one of a pristine probe as in Fig. 1(a), although this may be due, at least in part, to a different original shape. The cone in Fig. 6 appears sharp and uniformly coated, although the surrounding area is rather irregular. We note, in particular, that the metal coating is detached in two points at the base of the probe. In one case (black arrow) the coating has completely folded over. Nevertheless, the absence of any visible emission from probe A suggests that the adhesion between metallic coating and fiber is still good in the core region.

We consider that our experimental results can be explained, at least qualitatively, by taking into account the dif-

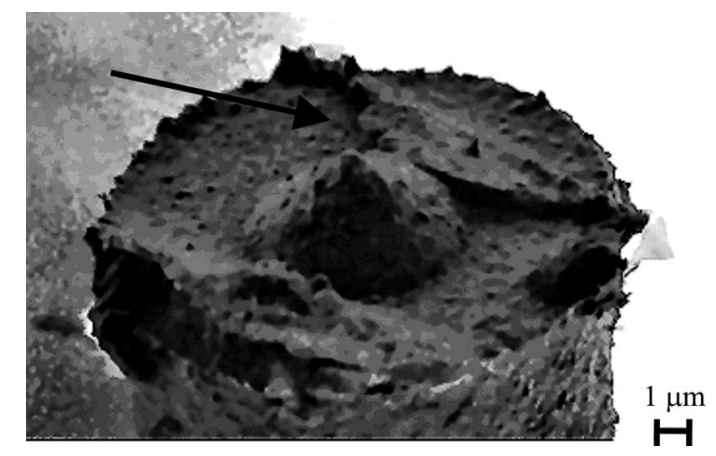

FIG. 6. SEM image of probe A (Jasco NPU series) acquired after the change of its thermomechanical behavior from shortening to elongation but still suitable for SNOM operation on the basis of its far-field transmission properties. 
ferent shapes and expansion coefficients of the glass fiber that constitutes the bulk of the SNOM probe and of the metallic coating. Whereas the former is essentially a glass cylinder some tens of microns in diameter and a few millimeters in length, which ends with a little cone $(10 \mu \mathrm{m}$ base diameter, $2 \mu \mathrm{m}$ height), the coating is a gold film that surrounds both the glass cylinder and the cone, only $\sim 150 \mathrm{~nm}$ thick, as depicted in Fig. 1(a). Laser absorption by the gold film is strongly localized in the conical region both because of the large absorption coefficient of gold (whose reflectance at normal incidence is only $25 \%$ at $325 \mathrm{~nm},{ }^{41}$ whereas optical transmission is virtually null) and because of multiple reflections at the cone faces. Furthermore, the thermal expansion of gold $\left(\alpha_{\text {gold }}=1.4 \times 10^{-5}{ }^{\circ} \mathrm{C}^{-1}\right.$ in bulk, at room temperature $)$ is nearly three times as large as that of silica $\left(\alpha_{\text {quartz }}=0.54\right.$ $\times 10^{-6}{ }^{\circ} \mathrm{C}^{-1}$ ) so that significant mechanical stress is expected to build up at the gold-fiber interface upon laser illumination that inevitably leads to heating and expansion of the gold film. When exceeding the adhesion force between the gold and the fiber, such stress may lead to a sliding of the gold film with respect to the glass support. A shortening of the total system could result, for example, from larger radial expansion of the cylinder base with respect to the coating that would pull back the metal surrounding the cone. The precise mechanism with which such effects are produced is not clear at the moment, and we note a full understanding of the processes at work requires careful three-dimensional, finite element simulation of the structures and their heating and expansion properties that goes beyond the scope of our work reported here. Such modeling should have the scope for clarifying several outstanding questions. For example, we find it indeed surprising that our data are consistent with a radial expansion of the cylinder greater than that of the gold on the base of the fiber, not least because both the temperature of the fiber and its expansion coefficient should be smaller than that of the Au film. However, there are further factors that also need to be taken into account, namely, the variation of the thermal expansion coefficient as a function of the Au thickness and dimensionality [the film is essentially two dimentional (2D) whereas the fiber is expected to behave essentially as a three-dimensional (3D) system] and the possible relaxation of the mechanical stress accumulated in the fiber cone during the probe-formation process that takes place via hard tapping. Another surprising aspect is that we are able to produce a shift of the tip position as large as $400 \mathrm{~nm}$ for a film thickness that is nominally only $100-150 \mathrm{~nm}$. This seems to point to a greater thickness of the $\mathrm{Au}$ in the apical region due either to the details of the $\mathrm{Au}$ evaporation or to those of the aperture formation process.

Before concluding, we wish, however, to note that clear evidence of the effects of mechanical stress can be found in Fig. 6, in the form of cracks and detachments of the metallic coating, one of which (black arrow) even shows the coating folding over itself while exposing part of the underlying fiber. As expected, this occurs more easily around the probe edges where the adhesion is reduced and the coating is thinner. Absence of radiation leakage in these conditions can be explained by the fact that such exposed areas belong to the cladding. We propose that such cracks are strictly related to

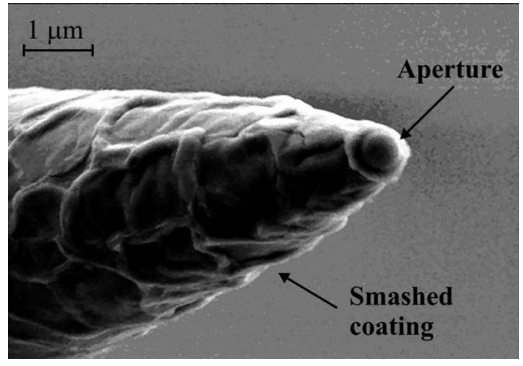

FIG. 7. SEM picture of a tube-etched aluminum-coated probe, subject to intense laser irradiation. The thermal damage arises through the melting of the metal film.

the "events" that we observe during high-power injection into the probes and that are accompanied by the probes reverting to the commonly observed "elongation" behavior in response to laser illumination.

Finally, it is interesting to compare the thermal behavior of SCE probes to pulled- or tube-etched sensors, ending with a long single conical taper, uniformly coated with aluminum. Figure 7 shows how the coupling of high laser powers $(>10 \mathrm{~mW}$ at $633 \mathrm{~nm})$ leads to the melting of the aluminum coating in a tube-etched probe. The film appears detached from the glass in proximity of the tip edge, and small folds are present everywhere due, again, to the local release of the stress induced by the different thermal expansion coefficients $\left(\alpha_{\text {aluminum }}=2.35 \times 10^{-5}{ }^{\circ} \mathrm{C}^{-1}\right)$. However, the lateral surface appears entirely damaged, up to distances of several microns from the tip apex.

\section{CONCLUSION}

We have studied the thermomechanical response of SNOM probes produced by selective chemical etching and coated by a gold film. We have observed that new probes shorten when UV light is coupled into them and that such effect is nonlinear as a function of inject power. Conversely, intensively used probes show a more conventional thermal elongation. SEM images show the formation of folds, due to the detachment of the metallic film from the glass surface, induced by the different thermal expansion coefficients. When strong thermal stresses occur at the cone base, we can observe the formation of cracks around the fiber core, leading to light leakage. Due to the peculiar geometry, the detachment starts at the fiber edges, forming folds that turn over themselves. This suggests that an improvement of the thermal resistance of this kind of probes could be obtained by strengthening the gold adhesion at the probe edges. This could be achieved, for example, by increasing the metal thickness at the edges or, alternatively, by interposing a further chromium layer between the glass and the gold, ${ }^{42}$ as is usually done in commercially available aluminum-coated probes.

\section{ACKNOWLEDGMENTS}

We are grateful to F. Intonti for her collaboration on the SEM observation of Fig. 7. One of the authors (A.A.) wishes to acknowledge the Galileo Galilei Graduate School of Pisa for supporting his stage at UCL and another author (P.G.G.) 
wishes to acknowledge the Venture Business Laboratory for supporting his stage at Kyoto. Partial financial support from the Italy-Japan bilateral Project 2A2 "Surface analysis and nanocharacterization of innovative materials by scanning probe microscopy techniques" is greatly acknowledged. One of the authors (F.C.) thanks the EPSRC and the Interdisciplinary Research Collaboration (Cambridge-UCL-Bristol) for financial and technical support and the Royal Society and the Wolfson Foundation for the award of a Laboratory Refurbishment grant.

${ }^{1}$ D. W. Pohl, W. Denk, and M. Lanz, Appl. Phys. Lett. 44, 651 (1984).

${ }^{2}$ A. Lewis, M. Isaacson, A. Harootunian, and A. Murray, Ultramicroscopy 13, 227 (1984).

${ }^{3}$ E. Betzig, M. Isaacson, and A. Lewis, Appl. Phys. Lett. 51, 2088 (1987)

${ }^{4}$ T. Saiki and K. Matsuda, Appl. Phys. Lett. 74, 2773 (1999).

${ }^{5}$ E. Betzig, J. K. Trautman, T. D. Harris, J. S. Weiner, and R. L. Kostelak, Science 251, 1468 (1991).

${ }^{6}$ R. L. Williamson and M. J. Miles, J. Appl. Phys. 80, 4804 (1996).

${ }^{7}$ G. A. Valaskovic, M. Holton, and G. H. Morrison, Appl. Opt. 34, 1215 (1995).

${ }^{8}$ T. Pangaribuan, K. Yamada, S. Jiang, H. Ohsawa, and M. Ohtsu, Jpn. J. Appl. Phys., Part 2 31, L1302 (1992).

${ }^{9}$ P. Lambelet, A. Sayah, M. Pfeffer, C. Philipona, and F. Marquis-Weible, Appl. Opt. 37, 7289 (1998).

${ }^{10}$ R. Stöckle, C. Fokas, V. Deckert, R. Zenobi, B. Sick, B. Hecht, and U. P. Wild, Appl. Phys. Lett. 75, 160 (1999).

${ }^{11}$ T. Yatsui, M. Kourogi, and M. Ohtsu, Appl. Phys. Lett. 73, 2090 (1998).

${ }^{12}$ A. Lazarev, N. Fang, Q. Luo, and X. Zhang, Rev. Sci. Instrum. 74, 3679 (2003).

${ }^{13}$ M. N. Islam, X. K. Zhao, A. A. Said, S. S. Mickel, and C. F. Valli, Appl. Phys. Lett. 71, 2886 (1997).

${ }^{14}$ T. Saiki, S. Monobe, M. Ohtsu, N. Saito, and J. Kusano, Appl. Phys. Lett. 68, 2612 (1996).

${ }^{15}$ S. Mononobe, M. Naya, T. Saiki, and M. Ohtsu, Appl. Opt. 36, 1496 (1997).

${ }^{16}$ R. Micheletto, N. Yoshimatsu, and S. Okazaki, Opt. Commun. 188, 11 (2001).

${ }^{17}$ J. A. Veerman, A. M. Otter, L. Kuipers, and N. F. Van Hulst, Appl. Phys.
Lett. 72, 3115 (1998).

${ }^{18}$ A. Bouhelier, J. Toquant, H. Tamaru, H.-J. Güntherodt, D. W. Pohl, and G. Schider, Appl. Phys. Lett. 79, 683 (2001).

${ }^{19}$ B. I. Yakobson and M. A. Paesler, Ultramicroscopy 57, 204 (1995).

${ }^{20}$ L. Novotny and C. Hafner, Phys. Rev. E 50, 4094 (1994).

${ }^{21}$ T. I. Kutznetsova, V. S. Lebedev, and A. M. Tsvelik, J. Opt. A, Pure Appl. Opt. 6, 338 (2004).

${ }^{22}$ A. Ambrosio, M. Allegrini, G. Latini, and F. Cacialli, Appl. Phys. Lett. 87, 033109 (2005).

${ }^{23}$ M. Stähelin, M. A. Bopp, G. Tarrach, A. J. Meixner, and I. ZschokkeGränacher, Appl. Phys. Lett. 68, 2603 (1996).

${ }^{24}$ Ch. Lienau, A. Richter, and T. Elsaesser, Appl. Phys. Lett. 69, 325 (1996).

${ }^{25}$ P. G. Gucciardi, M. Colocci, M. Labardi, and M. Allegrini, Appl. Phys. Lett. 75, 3408 (1999).

${ }^{26}$ A. H. La Rosa, B. I. Yakobson, and H. D. Hallen, Appl. Phys. Lett. 67, 2597 (1995).

${ }^{27}$ D. I. Kavaldjiev, R. Toledo-Crow, and M. Vaez-Iravani, Appl. Phys. Lett. 67, 2771 (1995).

${ }^{28}$ B. Biehler and A. H. La Rosa, Rev. Sci. Instrum. 73, 3837 (2002).

${ }^{29}$ J.-P. Mulet, K. Joulain, R. Carminati, and J.-J. Greffet, Appl. Phys. Lett. 78, 2931 (2001); G. Domingues, S. Volz, K. Joulain, and J.-J. Greffet, Phys. Rev. Lett. 94, 085901 (2005).

${ }^{30}$ G. Latini et al., Appl. Phys. Lett. 86, 011102 (2005).

${ }^{31}$ P. G. Gucciardi, S. Patanè, A. Ambrosio, M. Allegrini, A. D. Downes, G. Latini, O. Fenwick, and F. Cacialli, Appl. Phys. Lett. 86, 203109 (2005).

${ }^{32}$ R. Stevenson et al., Appl. Phys. Lett. 79, 833 (2001).

${ }^{33}$ R. Riehn, A. Charas, J. Morgado, and F. Cacialli, Appl. Phys. Lett. 82, 526 (2003).

${ }^{34}$ R. Riehn and F. Cacialli, J. Opt. A, Pure Appl. Opt. 7, S207 (2005).

${ }^{35}$ T. Inoue, F. Sato, and Y. Narita, Vib. Spectrosc. 35, 33 (2004).

${ }^{36}$ B. A. F. Puygranier and P. Dawson, Ultramicroscopy 85, 235 (2000).

${ }^{37}$ K. Karrai and W. Grober, Appl. Phys. Lett. 66, 1842 (1995).

${ }^{38}$ R. Micheletto, N. Yoshimatsu, M. Yokokawa, T. An, H. Lee, and S. Okazaki, Opt. Commun. 196, 47 (2001).

${ }^{39}$ E. Betzig, P. L. Finn, and J. S. Weiner, Appl. Phys. Lett. 60, 2484 (1992).

${ }^{40}$ Y. Narita (private communication).

${ }^{41}$ E. D. Palik, Handbook of Optical Constants of Solids (Academic, Orlando, 1985).

${ }^{42}$ H. G. Frey, F. Keilmann, A. Kride, and R. Guckenberger, Appl. Phys. Lett. 81, 5030 (2002) 